\title{
The Effect of Gd Impurities on the Physical Properties of Half-Metallic Ferromagnet $\mathrm{Co}_{2} \mathrm{MnSi}$
}

\author{
R. Grasin, E. Vinteler, A. Bezergheanu, C. Rusu, R. Pacurariu, i.G. Deac \\ AND R. TETEAN* \\ Faculty of Physics, Babes-Bolyai University, Kogalniceanu 1, RO-400084, Cluj Napoca, Romania
}

(Received January 26, 2010; in final form August 4, 2010)

\begin{abstract}
In this paper we report our experimental and theoretical studies on the effect of Gd impurity on the physical properties of the Heusler half-metallic ferromagnet $\mathrm{Co}_{2} \mathrm{MnSi}$. The analysis of the band structures of the doped alloy shows that the half-metallic properties are completely conserved if Gd substitutes Mn atoms. This effect is not determined by the spin-orbit interaction, but through the coupling between the $R(4 f)$ spin with the $\mathrm{Mn}(3 d)$ itinerant electron spins. We evaluate the strength of such a coupling by calculating, in an ab initio fashion, the total energy of $\mathrm{Co}_{16} \mathrm{GdMn}_{7} \mathrm{Si}_{8}$ compound for a parallel and antiparallel $f-d$ coupling. The obtained magnetic moments of Co or Mn sites are in good agreement with the experimental ones.
\end{abstract}

PACS numbers: 71.70.Ej, 71.55.Ak, 73.20.Hb

\section{Introduction}

The Heusler alloys containing Co and Mn are amongst the most heavily studied half-metallic ferromagnets for future applications in spintronics [1]. Ideal performance device would be obtained by incorporating a ferromagnet in which the conduction electrons are $100 \%$ spin polarized.

Half-metallicity was predicted by de Groot [2], when studying the band structure of half-Heusler NiMnSb alloy. Later on Kubler et al. [3] recognized that minority spin states in $\mathrm{Co}_{2} \mathrm{MnAl}$ nearly vanish, so the way in investigating the full-Heusler alloys having $\mathrm{Co}_{2} \mathrm{MnZ}(\mathrm{Z}=$ $\mathrm{Al}, \mathrm{Si}$, and $\mathrm{Ge}$ ) was opened. These alloys crystallize in a highly ordered $L 22_{1}$ structure, which belong to $F m \overline{3} m$ space group. $\mathrm{Co}_{2} \mathrm{MnSi}$ attracted particular interests because of its high Curie temperature $985 \mathrm{~K}$ and a gap of $0.4 \mathrm{eV}$ in the minority spin channel $[4,5]$. Structural and magnetic properties of $\mathrm{Co}_{2} \mathrm{MnSi}$ alloy were reported. A magnetic moment of $5.01 \mu_{\mathrm{B}} /$ f.u. was shown [6].

Sakuraba et al. [7] have fabricated magnetic tunnel junctions (MTJ) consisting of highly ordered $\mathrm{Co}_{2} \mathrm{MnSi}$ epitaxial bottom electrode, $\mathrm{Al}-\mathrm{O}$ tunnel barrier, and $\mathrm{Co}_{75} \mathrm{Fe}_{25}$ top electrode. A tunnelling magnetoresistance ratio (TMR) of $159 \%$ at low temperatures and a value of $70 \%$ at room temperature were determined. More recently, MTJ structures consisting of $\mathrm{Co}_{2} \mathrm{MnSi} / \mathrm{Al}-$ $\mathrm{O} / \mathrm{Co}_{2} \mathrm{MnSi}$ were fabricated, having a TMR ratio of $570 \%$, at $2 \mathrm{~K}$, the largest one reported to date for an $\mathrm{Al}-\mathrm{O}$

* corresponding author; e-mail: rote@phys.ubbcluj.ro amorphous tunneling barrier [8]. These experiments reveal the half-metallic ferromagnetic (HMF) character of $\mathrm{Co}_{2} \mathrm{MnSi}$ with a minority-spin band gap and a high decrease of TMR ratio with temperature [9].

Several results on half-metallic or semi-Heusler compounds showed that electronic correlations play a crucial role in depolarization effects by introducing nonquasiparticle (NQP) states just above the Fermi level [10-12]. Recently for the first time there has been shown the existence of NQP above the Fermi level in the minority spin channel, in $\mathrm{Co}_{2} \mathrm{MnSi}$ based tunnel junctions [13]. The existence of NQP states was proved by tunneling conductance measurements. The NQP picture suggests that it is necessary to pay attention to magnon excitations in order to improve the $\mathrm{Co}_{2} \mathrm{MnSi}$ based MTJ performances. For this it is necessary to modify the magnon excitations preserving in the same time the electronic properties (in principal the gap in the minority spin channel). Attema et al. have proposed to increase the magnetic anisotropy and thus the magnon gap by doping with rare-earth atoms in the half metallic material [14].

In this paper we analyse the effect of Gd substitution at $\mathrm{Mn}$ sites on the physical properties of $\mathrm{Co}_{2} \mathrm{MnSi}$ based alloy. The Gd $4 f$ electrons are not involved in chemical bonding. Total energy calculation allows us to evaluate the strength of the coupling between the $4 f$ rare-earth impurity spin and the $3 d$ manganese conduction electron spin.

\section{Experimental details}

The $\mathrm{Co}_{2} \mathrm{Mn}_{1-x} \mathrm{Gd}_{x} \mathrm{Si}$ compounds with $x=0,0.01$, 0.05 and 0.1 were prepared by arc melting of the con- 
stituent elements in a purified argon atmosphere. The ingots were remelted several times in order to ensure a good homogeneity. The samples were heat treated in vacuum, at $950{ }^{\circ} \mathrm{C}$, for 5 days. The crystal structure was checked by X-ray using a Bruker 8 XD diffractometer. Magnetic measurements were performed with a $12 \mathrm{~T}$ VSM magnetometer from Cryogenics in the temperature range $4.2-700 \mathrm{~K}$ and maximum external fields up to $12 \mathrm{~T}$. The spontaneous magnetizations, $M_{\mathrm{S}}$, were determined from magnetization isotherms according to approach to saturation law

$$
M=M_{\mathrm{S}}(1-b / H)+\chi_{0}^{\prime} H .
$$

We denoted by $b$ the coefficient of magnetic hardness and $\chi_{0}^{\prime}$ is a Pauli-type contribution. A SPECS multifunctional surface analysis system was used for X-ray photoelectron spectroscopy in order to check if Gd atoms occupied positions in the lattice sites or not.

Based on experimentally determined lattice constants, we have computed the band structures of $\mathrm{Co}_{2} \mathrm{Mn}_{0.875} \mathrm{Gd}_{0.125} \mathrm{Si}$ and $\mathrm{Co}_{2} \mathrm{MnSi}$ compounds using a supercell eight times greater than unit cell. A $d$-f-type model was used in the mean field approximation, in which the Mn $3 d$ and Gd $4 f$ states were described by LSDA $+U$ method, whereas the $3 d-4 f$ interaction was treated as perturbation. The mean-field Hamiltonian can be written in the form

$$
H \approx H_{\mathrm{LDA}+U}-J \sum \sigma_{i}^{3 d} S_{i+\delta}^{f},
$$

where the spin of the conduction electron at site $R_{i}$ is denoted by $\sigma_{i}^{3 d}$ and $S_{i+\delta}^{f}$ represents the spin of the $4 f$ shell at the $R_{i+\delta}$ site. LSDA $+U$ approach is based on the local spin density approximation (LSDA) to density functional theory (DFT) using exchange-correlation parameterization of von Barth and Hedin but is complemented with Hubbard- $U$ corrections treated in a mean field approximation. Our calculations use a full-potential linearized muffin-tin orbital (FP-LMTO) approach. This method uses an optimized basis set consisting of muffin-tin orbitals with smoothed Hankel functions as envelope functions [15]. The partially filled and strongly correlated localized $f$ orbitals were treated using the LSDA $+U$ method. In this method the Coulomb and exchange energy in the Hartree-Fock approximation of a chosen set of localized orbitals, here the $4 f$ states, are added to the usual local spin density functional and their orbital independent average is subtracted to avoid double counting. The LSDA $+U$ method is a physically motivated approach that attempts to incorporate the effects of important orbital specific local Coulomb interactions in strongly correlated electron systems while retaining the simplicity of local density approximation (LDA) calculations for real materials [16].

\section{Results and discussions}

The diffraction patterns for the samples with $x=0$ and $x=0.01$ are presented in Fig. 1. The X-ray analysis shows, in the limit of experimental errors, that the compounds are single phase with $\mathrm{BiF}_{3}$-type structure for the compounds with $x=0.00,0.01$ and 0.05 . In the case of the compound with $x=0.1$ it was found that in addition to $\mathrm{BiF}_{3}$-type structure, small quantity of CoMnSi phase is present. The alloys crystallize in a cubic structure having $F m \overline{3} m$ space group. The lattice parameter is $a=0.5654 \mathrm{~nm}$ and is very little affected by substitution, probably because of the small amount of Gd. In the case of compound with $x=0.1$ it was found that the line widths of the X-ray patterns are somewhat larger as compared with samples with less Gd, probably due to a small deformation of the lattice as the gadolinium content increases.
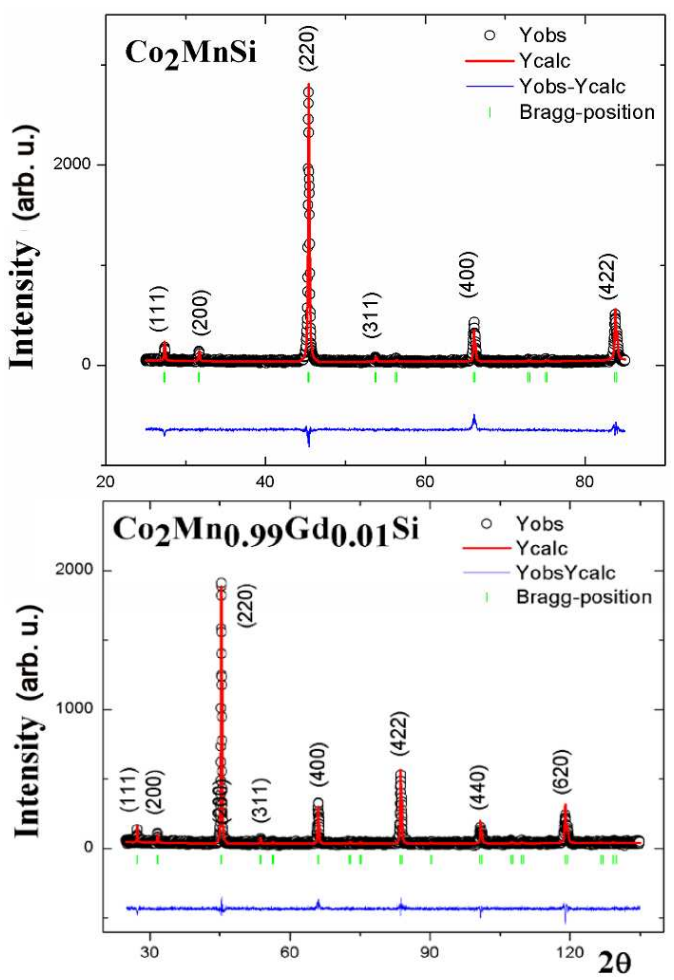

Fig. 1. Diffraction patterns for the compounds $\mathrm{Co}_{2} \mathrm{MnSi}$ and $\mathrm{Co}_{2} \mathrm{Mn}_{0.99} \mathrm{Gd}_{0.01} \mathrm{Si}$.

The Gd $4 f$ XPS spectra for the compound with $x=$ 0.01 is presented in Fig. 2. In the same figure the pure Gd spectra are shown. One can see that Gd spectra are changed by alloying which allows us to consider that Gd atoms occupy positions in the lattice and not interstitial. Further investigations are necessary in order to find the crystallographic position in which Gd enter.

The calculated densities of states (DOS), for $\mathrm{Co}_{16} \mathrm{Mn}_{7} \mathrm{GdSi}_{8}$ are presented in Fig. 3. In the case of $\mathrm{Co}_{16} \mathrm{Mn}_{7} \mathrm{GdSi}_{8}$ the calculations were done considering a ferromagnetic and respectively, antiferromagnetic coupling of $\mathrm{Gd}(4 f)$ and $\mathrm{Mn}(3 d)$ spins. The half-metallic state is stable in both cases with a band gap of $0.4 \mathrm{eV}$ magnitude. The analysis of the band structures of the doped alloy shows that the half-metallic properties are 


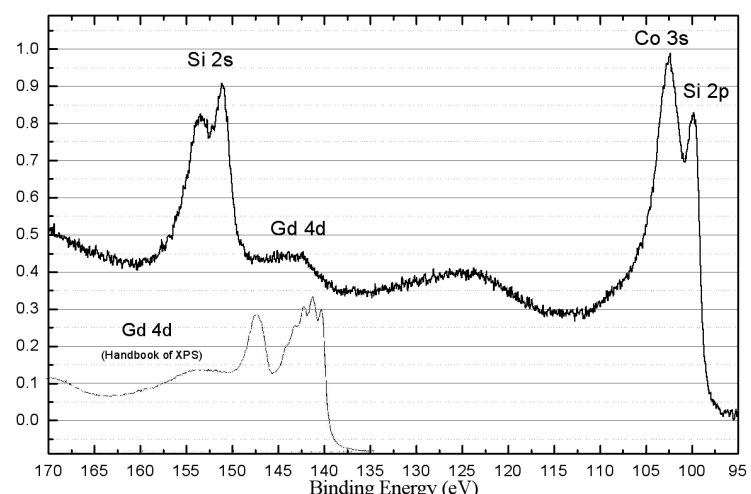

Fig. 2. Gd $4 f$ XPS spectra in $\mathrm{Co}_{2} \mathrm{Mn}_{0.99} \mathrm{Gd}_{0.01} \mathrm{Si}$. The XPS spectra of pure Gd are also shown.

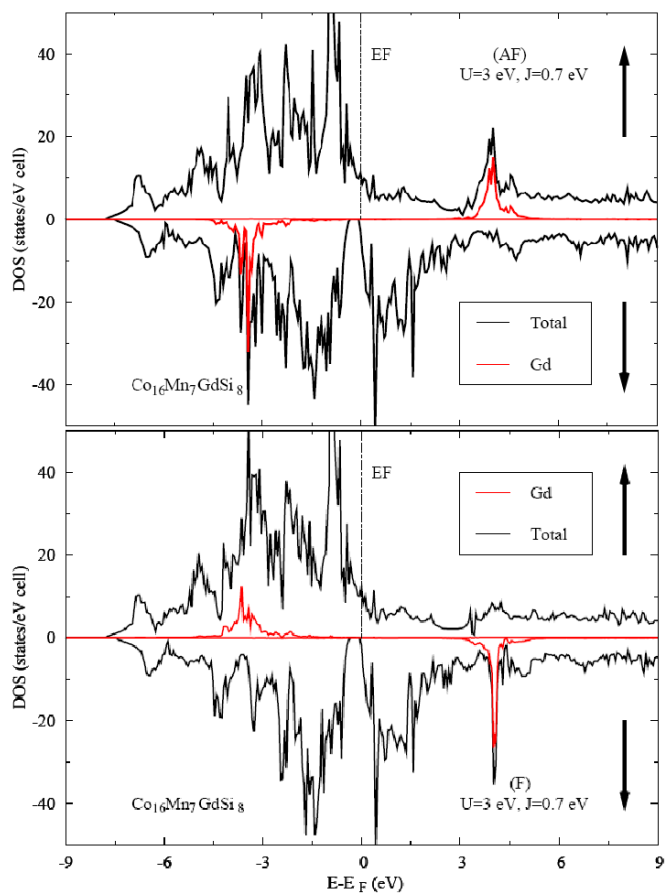

Fig. 3. Density of states for $\mathrm{Co}_{16} \mathrm{Mn}_{7} \mathrm{GdSi}_{8}$ compound in the cases of antiferromagnetic and ferromagnetic coupling.

completely conserved if gadolinium atoms substitute the manganese ones. This effect is not determined by the spin-orbit interaction, but through the coupling between the Gd $(4 f)$ spin with the Mn $(3 d)$ itinerant electron spins. We evaluate the strength of such a coupling by calculating, in an ab initio fashion, the total energy of $\mathrm{Co}_{16} \mathrm{Mn}_{7} \mathrm{GdSi}_{8}$ compound for a parallel and antiparallel $f-d$ coupling. Given the geometry of the cell, the lanthanide substitution is realized in the fcc Mn sublattice, so 12 pairs of $\mathrm{Gd}(4 f)-\mathrm{Mn}(3 d)$ are formed. As a consequence the $f-d$ coupling constant was calculated as the $E_{\text {Ferro }}-E_{\text {Antiferro }}$ energy corresponding to a pair and has values of $54 \mathrm{~K}$ for $U=7 \mathrm{eV}, J=0.7 \mathrm{eV}$ and
$105 \mathrm{~K}$ for $U=3 \mathrm{eV}, J=0.7 \mathrm{eV}$. One can see that the minimum energy is in the case of an antiparallel coupling between $\mathrm{Mn}(3 d)$ and $\mathrm{Gd}(4 f)$ spins as it is expected for heavy rare-earths $-3 d$ transition metals compounds [17]. We expect that at temperatures lower than that calculated for the exchange coupling the gadolinium substitution could affect the magnonic excitations leaving the half-metallic gap unmodified. Table shows the atomic magnetic moments for the pure compound data obtained for the Gd-doped samples. A modification of Co moment is shown, as result of doping effect. On the other hand, a small negative moment was found on silicon due to the hybridization effects. The calculated magnetic moments are $5.00 \mu_{\mathrm{B}} /$ f.u. for $\mathrm{Co}_{2} \mathrm{MnSi}$ and $5.35 \mu_{\mathrm{B}} /$ f.u. (F coupling), respectively $3.59 \mu_{\mathrm{B}} /$ f.u. (AF coupling) for $\mathrm{Co}_{2} \mathrm{Mn}_{0.875} \mathrm{Gd}_{0.125} \mathrm{Si}$ compound compound $(U=3 \mathrm{eV}$, $J=0.7 \mathrm{eV})$.
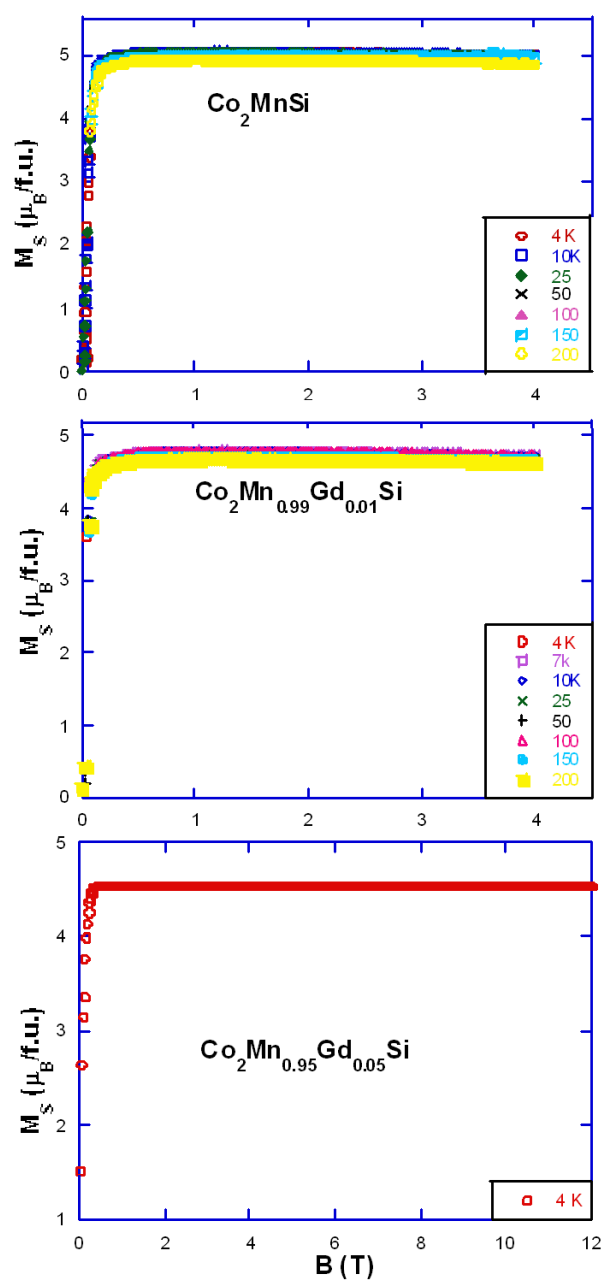

Fig. 4. Magnetizations isotherms at different temperatures.

The magnetization isotherms at different temperatures are shown in Fig. 4. One can see that the samples are saturated in external field of $3 \mathrm{~T}$. From the magnetization isotherms at $4 \mathrm{~K}$ the spontaneous magneti- 
zations were determined. Considering that the magnetic moments per atom of $\mathrm{Co}, \mathrm{Mn}, \mathrm{Gd}$ and $\mathrm{Si}$ are the same, like that obtained in the $\mathrm{Co}_{16} \mathrm{Mn}_{7} \mathrm{GdSi}_{8}$ compound, we have calculated the magnetic moments for $\mathrm{Co}_{2} \mathrm{Mn}_{0.99} \mathrm{Gd}_{0.01} \mathrm{Si}$ and $\mathrm{Co}_{2} \mathrm{Mn}_{0.95} \mathrm{Gd}_{0.05} \mathrm{Si}$. Magnetic moments values of $4.47 \mu_{\mathrm{B}} /$ f.u. $(x=0.01)$, respectively $4.06 \mu_{\mathrm{B}} /$ f.u. $(x=0.05)$, were obtained for the data cal- culated with $U=7 \mathrm{eV}$ and $4.74 \mu_{\mathrm{B}} /$ f.u. $(x=0.01)$ respectively $4.35 \mu_{\mathrm{B}} /$ f.u. $(x=0.05)$, for the data calculated with $U=3$. There is good agreement between the calculated values in case of antiferomagnetic coupling and experimental values: $5.07 \mu_{\mathrm{B}} /$ f.u. for $x=0.00,4.75 \mu_{\mathrm{B}} /$ f.u. $(x=0.01)$ and $4.53 \mu_{\mathrm{B}} /$ f.u. $(x=0.05)$. The best agreement is for the values obtained from theoretical calculations with $U=3 \mathrm{eV}$.

TABLE

Calculated magnetic moments for $\mathrm{Co}_{16} \mathrm{Mn}_{8} \mathrm{Si}_{8}$ and $\mathrm{Co}_{16} \mathrm{Mn}_{7} \mathrm{GdSi}_{8}$ compounds, respectively, in case of ferromagnetic coupling $(\mathrm{F})$ and antiferromagnetic coupling (AF) for different $U J$ values.

\begin{tabular}{l|c|c|c|c|c}
\hline \hline$U[\mathrm{eV}], J[\mathrm{eV}]$ & & \multicolumn{2}{|c|}{$7,0.7$} & \multicolumn{2}{c}{$3,0.7$} \\
\hline \multicolumn{1}{c|}{ Coupling } & & $\mathrm{F}$ & $\mathrm{AF}$ & $\mathrm{F}$ & $\mathrm{AF}$ \\
\hline $\mathrm{Co}_{1}\left[\mu_{\mathrm{B}} /\right.$ atom $]$ & 1 & 0.793 & 0.802 & 1.099 & 1.095 \\
$\mathrm{Co} 2\left[\mu_{\mathrm{B}} /\right.$ atom $]$ & 1 & 0.793 & 0.802 & 0.808 & 0.787 \\
$\mathrm{Mn}\left[\mu_{\mathrm{B}} /\right.$ atom] & 3.03 & 3.036 & 3.038 & 3.036 & 3.038 \\
$\mathrm{Gd}\left[\mu_{\mathrm{B}} /\right.$ atom] & - & 6.947 & -7.125 & 6.837 & -7.04 \\
$\mathrm{Si}\left[\mu_{\mathrm{B}} /\right.$ atom] & -0.03 & -0.064 & -0.068 & -0.064 & -0.067 \\
$\mathrm{M}\left[\mu_{\mathrm{B}} / 8\right.$ f.u. $]$ & 40.00 & 42.89 & 28.882 & 42.916 & 28.82311187 \\
$\mathrm{M} / 8\left[\mu_{\mathrm{B}} /\right.$ f.u. $]$ & 5.00 & 5.36125 & 3.61025 & 5.3645 & 3.602888984 \\
\hline$E_{\text {tot }}[\mathrm{eV}]$ & -8458.7589 & -87887.4634 & -87887.4675 & -87887.4971 & -87887.5051 \\
\hline$E_{\text {Ferro }}-E_{\text {Antiferro }}[\mathrm{K}]$ & \multicolumn{2}{|c|}{54.1190485} & & \multicolumn{2}{c}{105.508336} \\
\hline
\end{tabular}

\section{Conclusions}

We conclude that Gd has entered into the transition metal sublattice in $\mathrm{Co}_{2} \mathrm{MnSi}$. The electronic structure calculations suggest that the half-metallicity is preserved when Gd substitute Mn sites. The best agreement with experimental data was obtained for the calculated values obtained by considering $U=3 \mathrm{eV}$. At temperatures lower than that calculated for the exchange coupling $(105 \mathrm{~K}$ for $U=3 \mathrm{eV}$ ) the gadolinium substitution could affect the magnonic excitations leaving the half-metallic gap unmodified. Further investigations are necessary.

\section{Acknowledgments}

This work was supported by the Ministry of Education and Research of Romania, grant CNCSIS-ID-2578/2008, No. 565 .

\section{References}

[1] M.I. Katsnelson, V.Yu. Irkhin, L. Chioncel, A.I. Lichtenstein, R.A. de Groot, Rev. Mod. Phys. 80, 315 (2008).

[2] R.A. de Groot, F.M. Mueller, P.G. van Engen, K.H.J. Buschow, Phys. Rev. Lett. 50, 2024 (1983).

[3] J. Kubler, A.R. Williams, C.B. Sommers, Phys. Rev. B 28, 1745 (1983).

[4] S. Fuji, S. Sugimura, S. Ishida, S. Asano, J. Phys., Condens. Matter 2, 8583 (1990).

[5] P.J. Brown, K.-U. Neumann, P.J. Webster, K.R.A. Ziebeck, J. Phys., Condens. Matter 12, 1827 (2000).

[6] H. Ido, J. Magn. Magn. Mater. 54-57, 937 (1986).
[7] Y. Sakuraba, T. Miyakoshi, M. Oogane, Y. Ando, A. Sakuma, T. Miyazaki, H. Kubota, Appl. Phys. Lett. 89, 052508 (2006)

[8] Y. Sakuraba, M. Hattori, M. Oogane, Y. Ando, H. Kato, A. Sakuma, T. Miyazaki, H. Kubota, Appl. Phys. Lett. 88, 192508 (2006).

[9] Y. Sakuraba, M. Hattori, M. Oogane, H. Kubota, Y. Ando, A. Sakuma, T. Miyazaki, J. Phys. D 40, 1221 (2007).

[10] L. Chioncel, M. I. Katsnelson, R. A. de Groot, A. I. Lichtenstein, Phys. Rev. B 68, 144425 (2003).

[11] L. Chioncel, E. Arrigoni, M.I. Katsnelson, A.I. Lichtenstein, Phys. Rev. Lett. 96, 137203 (2006).

[12] L. Chioncel, Ph. Mavropoulos, M. Lezaic, S. Blügel, E. Arrigoni, M.I. Katsnelson, A.I. Lichtenstein, Phys. Rev. Lett. 96, 197203 (2006).

[13] L. Chioncel, Y. Sakuraba, E. Arrigoni, M.I. Katsnelson, M. Oogane, Y. Ando, T. Miyazaki, E. Burzo, A.I. Lichtenstein, Phys. Rev. Lett. 100, 086402 (2008).

[14] J.J. Attema, C.M. Fang, L. Chioncel, G.A. de Wijs, A.I. Lichtenstein, R.A. de Groot, J. Phys., Condens. Matter 16, S5517 (2004).

[15] P. Larson, W.R.L. Lambrecht, Phys. Rev. B 74, 085108 (2006).

[16] S.G. Louie, APS March Meeting, 13-17 March 2006, Baltimore, USA, unpublished.

[17] E. Burzo, A. Chelkowski, H.R. Kirchmayr, Landolt Börnstein Handbook, Vol. 19d2, Springer-Verlag, Heidelberg 1990, p. 130. 